\title{
Stress-Induced Anhedonia in Mice is Associated with Deficits in Forced Swimming and Exploration
}

\author{
Tatyana Strekalova', Rainer Spanagel', Dusan Bartsch', Fritz A Henn*,' and Peter Gass' \\ 'Central Institute of Mental Health, University of Heidelberg, Mannheim, Germany
}

\begin{abstract}
In order to develop a model for a depression-like syndrome in mice, we subjected male C57BL/6 mice to a 4-week-long chronic stress procedure, consisting of rat exposure, restraint stress, and tail suspension. This protocol resulted in a strong decrease in sucrose preference, a putative indicator of anhedonia in rodents. Interestingly, predisposition for stress-induced anhedonia was indicated by submissive behavior in a resident-intruder test. In contrast, most mice with nonsubmissive behavior did not develop a decrease in sucrose preference and were regarded as nonanhedonic. These animals were used as an internal control for stress-induced behavioral features not associated with the anhedonic state, since they were exposed to the same stressors as the anhedonic mice. Using a battery of behavioral tests after termination of the stress procedure, we found that anhedonia, but not chronic stress per se, is associated with key analogues of depressive symptoms, such as increased floating during forced swimming and decreased exploration of novelty. On the other hand, increased anxiety, altered locomotor activity, and loss of body weight were consequences of chronic stress, which occurred independently from anhedonia. Thus, behavioral correlates of stress-induced anhedonia and of chronic stress alone can be separated in the present model.

Neuropsychopharmacology (2004) 29, 2007-2017, advance online publication, 2 I July 2004; doi: I 0. I 038/sj.npp. I 300532
\end{abstract}

Keywords: anhedonia; chronic stress; sucrose preference; depression model; forced swimming; mouse

\section{INTRODUCTION}

Anhedonia, a decreased ability to experience pleasures, is a core symptom of human depression (Hamilton, 1967; Klein, 1974; Kessler et al, 1994). It has been proposed that hedonic deficits can also be induced in rodents by chronic stress (Katz, 1981; Willner et al, 1987; D'Aquila et al, 1997; Harkin et al, 2002). Thus, Katz and co-workers subjected rats to a 21-day-long stress protocol, including electric shocks, immobilization, swimming in cold water, and other strong stimuli, which caused a decrease of sucrose intake that was interpreted as a sign of an hedonic deficit (Katz, 1981). To obtain a closer analogy to the human situation, Willner and his group used milder stressors such as soiled cage stress, presence of novel objects, restricted access to food, etc, and extended the stress exposure up to 3 months that produced a longer lasting decrease in sucrose consumption (Willner et al, 1987; Willner, 1991, 1997).

Besides a decrease in sucrose consumption, chronic stress in rodents has been reported to evoke a variety of physiological abnormalities that are also observed in human

\footnotetext{
*Correspondence: Professor FA Henn, Central Institute of Mental Health, J 5, Mannheim D-68I59, Germany, Tel: +49 62I 1703738 , Fax: + 49621 1703 760, E-mail: henn@zi-mannheim.de Received 5 June 2003; revised 7 April 2004; accepted 8 June 2004 Online publication: 23 June 2004 at http://www.acnp.org/citations/ Npp06230403253/default.pdf
}

depression, for example, reduced latency and increased duration of REM sleep (Moreau et al, 1995; Cheeta et al, 1997), diminished sexual activity (D'Aquila et al, 1994; Brotto et al, 2001), increased corticosterone levels (Katz, 1981), and disturbed circadian rhythms (Stewart et al, 1990; Solberg et al, 1999). However, in these animal studies, chronic stress did not induce other depressive symptoms such as increased floating in the forced swim test and diminished exploration activity (Katz, 1982; D'Aquila et al, 1994; Hata et al, 1995; Harris et al, 1997). Thus, in animals, depression-like behavioral features in relation to hedonic deficits are currently not well characterized.

We describe here a new chronic stress procedure in mice that induces anhedonia only in a subgroup of stressed animals, while another group of stressed animals do not show hedonic deficits and can serve as an internal control for the stress effects not associated with anhedonia. Since clinical studies in depressed patients indicate pre-existing behavioral features that predispose to the development of depression and anhedonia (Henn, 1980; Kessler et al, 1994), we investigated which interindividual differences in initial behavior in mice correlated with the vulnerability to stressinduced anhedonia. Earlier ethological studies have revealed similarities in the behavioral characteristics of animals with a submissive type of social behavior and helpless/defeated animals in behavioral models of depression (Raab et al, 1986; Kudryavtseva et al, 1991; Pare and Redei, 1993; Blanchard et al, 1993; Vollmayr and Henn, 
2001). Furthermore, loss of social rank in rats in repeated defeat paradigm has been followed by decrease in sucrose consumption (Willner et al, 1995). Therefore, in our study we used mice in which social status was defined before stress exposure as submissive or nonsubmissive.

\section{EXPERIMENTAL METHODS}

\section{Animals and Housing Conditions}

Adult C57BL/6N male mice (age: 4 months; average body weight: $31.7 \pm 0.9 \mathrm{~g}$ ) were purchased from Charles River (Sulzfeld, Germany). At 2 weeks before the behavioral experiments, mice were housed single caged under a reverse 12:12 h light-dark cycle (lights on: 1800) in standard laboratory conditions $\left(22 \pm 1{ }^{\circ} \mathrm{C}, 55 \%\right.$ humidity, food and water ad libitum). Male CD1 mice, used as intruders in a resident-intruder test, were purchased from the same supplier and kept in the same animal room, except that they were group housed with five individuals per cage. All experiments had been approved by the local governmental body of animal care and welfare.

\section{Experimental Design}

We selected mice with either submissive or nonsubmissive behavior using a resident-intruder test (see behavioral tests). Mice with nondefinable social status were not used in the experiments. Subsequently, 26 mice (eight submissive and 18 nonsubmissive animals) were assigned to a group subjected to chronic stress. A total of 12 mice (five submissive and seven nonsubmissive animals) formed a nonstressed control group. At 1 week before the chronic stress procedure, all mice were studied in the following tests: open field, novel cage, new object exploration, Omaze, and dark/light box (see behavioral tests). Body weight, baseline preference to a $1 \%$ sucrose solution, and sucrose intake were also determined (see behavioral tests).

The chronic stress protocol consisted of three different stressors (see chronic stress procedure) and was applied until a decrease in sucrose preference developed in the majority of the tested population. Four mice died during the different stages of the experiment (two submissive mice died between $3 \frac{1}{2}$ and 4 weeks of stress procedure; two nonsubmissive individuals died after termination of the stress protocol). None of the nonstressed control animals died. The sucrose consumption test was performed $3,3 \frac{1}{2}$, and 4 weeks after beginning of the stress procedure and 1 day, 1,2 and 3 weeks after its termination (see behavioral tests). During the first week after termination of the stress procedure, all mice were tested in the open field, activity boxes, forced swim test, new object exploration test, novel cage, O-maze, and dark/light box.

\section{Chronic Stress Procedure}

The chronic stress procedure consisted of exposure to rat, restraint stress, and tail suspension. Stressors were applied in following sequence: days 1-7, exposure to a rat; days $8-10$, restraint stress; days $11-17$, exposure to rat; days $18-22$, tail suspension; days $23-25$, restraint stress; and days 26-28, tail suspension.
Exposure to rat. Mice were individually introduced into small cages of size $22 \times 8.5 \times 14 \mathrm{~cm}^{3}$, which were then placed for $15 \mathrm{~h}$ (1800-0900) into a rat home cage of size $38 \times 22 \times 15 \mathrm{~cm}^{3}$, containing a rat. Holes (diameter $0.7 \mathrm{~cm}$ ) made in the front wall of the mouse cage assured an olfactory contact to the predator. Water and food were available at libitum. The rest of the day (0900-1800) mice were housed in their home cages in the same experimental room.

Restraint stress. Animals were placed inside a plastic tube (internal diameter $26 \mathrm{~mm}$ ) for $2 \mathrm{~h}$ during the dark phase of the light cycle and kept in a dark experimental room.

Tail suspension stress. Mice were submitted to the tail suspension procedure by hanging them by their tails in a tail suspension system (Bioseb, France) for $6 \mathrm{~min} /$ day. The procedure was carried out during the dark phase of the animals' light cycle.

\section{Behavioral Tests}

General conditions. Each behavioral test was carried out by the same person between 0930 and 1600. All mice were tested each day using one test, which was recorded on videotape. Animals were allowed to adapt to the experimental room for at least $1 \mathrm{~h}$ before testing.

Resident-intruder test. The testing procedure was performed as described elsewhere (Krsiak, 1975; Krsiak et al, 1998). In this paradigm, only animals for which the social behavior (submissive or non-submissive) was definable were selected to form experimental groups. The C57BL/6 mice were placed individually as a resident in an observation cage $\left(30 \times 60 \times 30 \mathrm{~cm}^{3}\right)$ for $30 \mathrm{~min}$. Thereafter, a male CD1 mouse, which was housed in the group before the test, was introduced as an intruder to the same cage and left with the resident mouse for $8 \mathrm{~min}$. During observation period, resident and intruder mice displayed either aggressive, submissive, or neutral social exploratory behavior. Complete lack of attacks toward the partner, accompanied by specific 'submissive' postures, escape, and defense was regarded as submissive type of social behavior. Initiation of attacks toward the partner and fighting back in response to attacks were categorized as nonsubmissive behavior. Some pairs of animals did not exhibit behavioral confrontation, but instead showed no social interaction or social exploratory behavior; these mice were regarded as nondefinable for their social status and were not included to experimental groups. In nonsubmissive animals latency of the first attack and number of attacks were scored.

Forced swim test. Mice were introduced to a transparent pool $\left(20 \times 35 \times 15 \mathrm{~cm}^{3}\right)$ filled with warm water $\left(30^{\circ} \mathrm{C}\right.$, height $9.5 \mathrm{~cm}$ ) and lit by red light for $6 \mathrm{~min}$. Parameters of floating behavior, defined as the absence of directed movements of animals' head and body were estimated. Latency to begin floating was scored as time between introduction of a mouse into the pool and the very first moment of complete immobility of entire animal body, irrespectively of the duration of the first floating episode. The total time spent floating was scored during the first $2 \mathrm{~min}$ of the test. 
New object exploration test. Mice were allowed to explore a new object for $15 \mathrm{~min}$ in an area $18 \times 25 \mathrm{~cm}^{2}$, surrounded by plastic walls. Illumination intensity was $5 \mathrm{~lx}$. The object, with a complex texture surface (artificial flower, $2.5 \times 2.5 \times 4 \mathrm{~cm}^{3}$ ), was fixed to the center of exploration area. The total duration of time spent exploring the object was scored by visual observation from video records off-line.

Novel cage test. The novel cage test was performed to assess exploration of a new environment. Mice were introduced into a standard plastic cage filled with fresh sawdust. The number of exploratory rearings was counted under red light during a 5 min period by visual observation.

Elevated O-maze. The maze consisted of a black circular path (runway width $5.5 \mathrm{~cm}$, diameter $46 \mathrm{~cm}$ ) that was covered with fleece paper and placed $20 \mathrm{~cm}$ above the floor. Illumination intensity was $5 \mathrm{~lx}$. Two opposing compartments were protected by walls of gray polyvinyl chloride (height $10 \mathrm{~cm}$ ). Mice were introduced to one of the two closed compartments. Number of exits to the anxietyrelated open compartments of the maze and total duration of time spent therein were scored during a 5 min observation period.

Dark-light box. The dark/light box consisted of two plexiglass compartments, one black/dark $\left(15 \times 20 \times 25 \mathrm{~cm}^{3}\right)$ and one lit $\left(30 \times 20 \times 25 \mathrm{~cm}^{3}\right)$, connected by a tunnel. Mice were placed into the dark compartment, from where they could visit the lit box, illuminated by light of $50 \mathrm{~lx}$ intensity. Total duration of time spent in the lit box and number of visits to this anxiety-related compartment were scored by visual observation over $5 \mathrm{~min}$.

Open field test. Open field arenas $\left(50 \times 50 \times 40 \mathrm{~cm}^{3}\right)$ were made of wood covered by white plastic. Boxes were evenly illuminated with white light ( 25 or $5 \mathrm{~lx}$ ) using a shadow-free illumination system. Horizontal locomotor activity (total distance moved) was measured over a $15 \mathrm{~min}$ period using a video-tracking system (Ethovision Program 1.95, Noldus, Wageningen, The Netherlands).

Activity boxes. Mice were placed for $10 \mathrm{~min}$ into dark locomotor activity boxes $\left(26 \times 26 \times 39 \mathrm{~cm}^{3}\right.$; Coulbourn Instruments, Allentown, PA, USA). Horizontal movements were scored automatically by a line of red beam cells situated $2 \mathrm{~cm}$ above the bottom using Truscan software (Coulbourn).

Sucrose consumption test. During this test, mice were given, for $24 \mathrm{~h}$, a free choice between two bottles, one with $1 \%$ sucrose solution and another with tap water. To prevent possible effects of side preference in drinking behavior, the position of the bottles was switched after $12 \mathrm{~h}$. No previous food or water deprivation was applied before the test. The consumption of water and sucrose solution was estimated simultaneously in control and experimental groups by weighing the bottles. The sucrose intake was calculated as an amount of consumed sucrose in mg per gram body weight. The preference for sucrose was calculated as a percentage of consumed sucrose solution of the total amount of liquid drunk. A decrease of sucrose preference below $65 \%$, measured at 4 weeks of continuous stress application, was taken as the criterion for anhedonia. This criterion was based on the fact that none of the control mice exhibited $\leqslant 65 \%$ preference for sucrose at that time point of the experiment. In addition, mice with a sucrose preference $\leqslant 65 \%$ in other models had shown features of anhedonia and depression, such as an increased threshold of intracranial self-stimulation and sleep disturbances (Vogel et al, 1990; Moreau et al, 1992; Willner, 1997). Mice that matched this definition were assigned to the anhedonic group. The rest of the stressed animals were considered to be nonanhedonic. Mice were assigned to nonanhedonic or anhedonic groups before further testing or data analysis.

\section{Statistical Analysis}

Data were analyzed with a statistical software package (Systat 10.01, Chicago, IL). Since the behavioral parameters analyzed were not normally distributed, nonparametric tests were used. Independent data sets were analyzed by the Kruskal-Wallis test, followed by the Mann-Whitney $U$-test. Repeated measurements were evaluated by the Wilcoxon test. Qualitative data were analyzed by the Fischer's exact test (exact probability for contingency table). The level of confidence was set at $95 \%(P<0.05)$.

\section{RESULTS}

\section{Chronic Stress Induces a Lasting Decrease of Sucrose Preference in a Subgroup of Mice}

Initially, all three groups of mice-anhedonic, nonanhedonic, and controls - had a similar preference for sucrose and consumption of sucrose solution and water (see Table 1). Our 4-week stress procedure caused a significant drop of sucrose preference in the majority of stressed mice compared to the values before stress and to the means of the nonstressed control group (Figure 1). Of stressed mice, $61 \%$ showed a sucrose preference below $65 \%$ and were defined as anhedonic. The mean preference for sucrose in this group was $51.2 \pm 3.8 \%$. In total, $39 \%$ of stressed animals demonstrated a sucrose preference over $65 \%$ and were regarded as nonanhedonic. The mean preference for sucrose shown by nonanhedonic mice $(75.1 \pm 1.7 \%)$ did not statistically differ from that of nonstressed controls $(78.8 \pm 1.1 \% ; p=0.518$ Figure $1 \mathrm{a}$ and $\mathrm{b})$. Mice defined as anhedonic showed significantly diminished sucrose preference compared to control mice when measured 1 day after stress $(p<0.001), 1$ week ( $p=0.001$; Figure $1 \mathrm{a}$ and $\mathrm{b})$, and 2 weeks after termination of chronic stress $(p=0.041$; Figure 2$)$. At the time point 3 weeks after termination of stress, all groups of mice had similar preference for sucrose $(p>0.05)$. Anhedonic mice showed significantly diminished amount of consumed sucrose solution and of sucrose intake per body weight compared to control mice when measured 1 day after stress ( $p=0.002$ and 0.003 , respectively) and 1 week $(p=0.003$ and 0.003 , respectively; Figures $1 \mathrm{c}$ and 3$)$. In contrast, the absolute intake of sucrose solution and of sucrose intake calculated per body weight measured in nonanhedonic mice did not statistically differ from that of 
Table I Baseline Behavior of Control, Nonanhedonic, and Anhedonic Mice

\begin{tabular}{lcccccc}
\hline & $\begin{array}{c}\text { OF test, 25 Ix: } \\
\text { total distance } \\
\text { moved (cm) }\end{array}$ & $\begin{array}{l}\text { Novel cage: } \\
\text { rearings (n) }\end{array}$ & $\begin{array}{c}\text { Duration of } \\
\text { exploration of } \\
\text { new object (s) }\end{array}$ & $\begin{array}{c}\text { O-maze: time } \\
\text { spent in open } \\
\text { arms (s) }\end{array}$ & $\begin{array}{c}\text { Dark/light: time } \\
\text { spent in lit box } \\
\mathbf{( s )}\end{array}$ & $\begin{array}{c}\text { Sucrose preference (\%)/ } \\
\text { Sucrose intake (m)/Water } \\
\text { intake (m) }\end{array}$ \\
\hline Control & $6863 \pm 419$ & $76.4 \pm 7.2$ & $81.6 \pm 18.2$ & $51.5 \pm 9.2$ & $73.1 \pm 13.2$ & $80.3 \pm 1.9 / 4.0 \pm 0.31 / 1.0 \pm 0.16$ \\
Nonanhedonic & $6927 \pm 583$ & $75.0 \pm 5.0$ & $90.1 \pm 15.2$ & $46.1 \pm 12.8$ & $69.8 \pm 17.2$ & $77.9 \pm 1.5 / 3.8 \pm 0.34 / 1.07 \pm 0.19$ \\
Anhedonic & $6421 \pm 220$ & $83.1 \pm 5.2$ & $88.1 \pm 19.2$ & $58.0 \pm 9.6$ & $81.4 \pm 10.9$ & $77.3 \pm 1.8 / 3.76 \pm 0.30 / 1.09 \pm 0.11$ \\
\hline
\end{tabular}

Data are shown as mean \pm SEM.

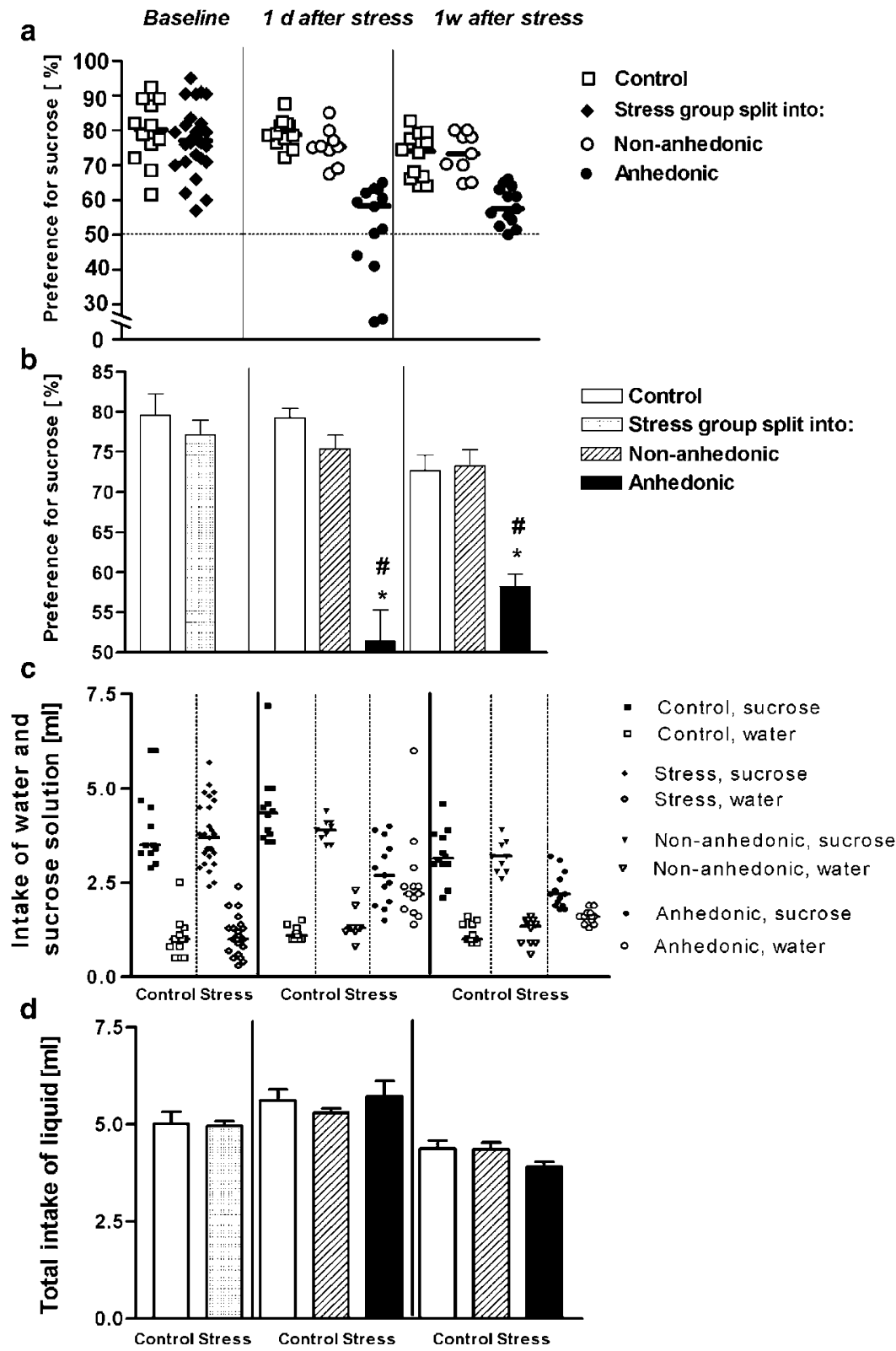

Figure I Chronic stress leads to a decrease in sucrose preference and absolute intake. (a) Chronic stress causes a drop of sucrose preference, measured I day after stress termination, in a subgroup of mice. According to the criterion of $65 \%$ preference for sucrose solution over water (see the text), the group of stressed mice split into anhedonic and nonanhedonic subgroups. At I week after termination of the chronic stress procedure, the sucrose preference was decreased in mice from the anhedonic group (bars indicate medians of the groups). (b) After stress, the mean sucrose preference of mice, which was defined as anhedonic was significantly lower compared to control and nonanhedonic groups. (* $p<0.05$ vs control group and ${ }^{\#}<<0.05$ vs nonanhedonic group). There were no significant differences between nonanhedonic and control groups in any of the measurements ( $p>0.05$ ). (c) Individual data show that chronic stress caused decrease of intake of sucrose solution in a subgroup of mice I day and I week after stress termination (bars indicate medians of the groups). (d) Total intake of liquid (water plus sucrose solution) did not change between the groups ( $p>0.05$ ). 


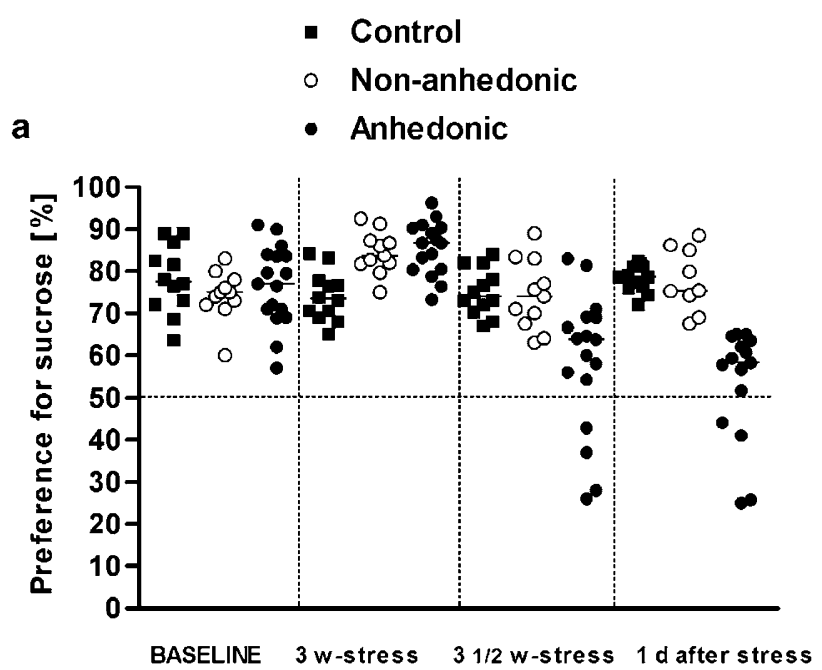

b

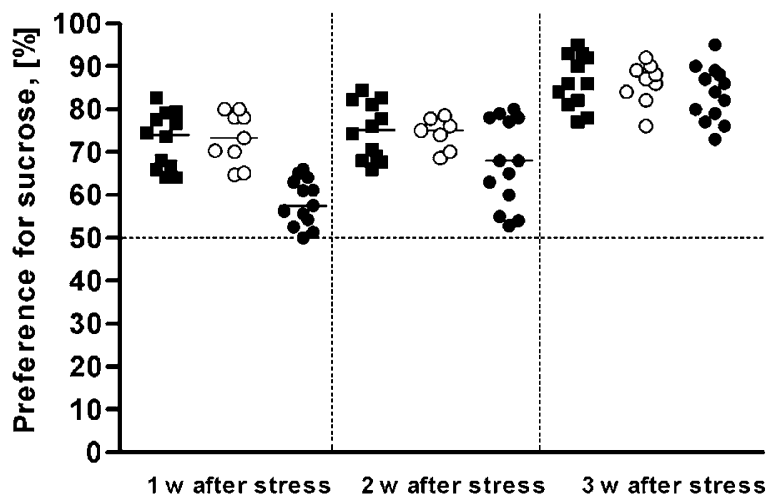

Figure 2 Dynamic of sucrose preference during experiment. (a) Anhedonic and nonanhedonic groups of mice did not differ in sucrose preference before stress (at baseline measurement) and at the time point 3 weeks after beginning of stress exposure. In anhedonic group, chronic stress caused a drop in sucrose preference measured $3 \frac{1}{2}$ weeks after beginning of stress exposure and I day after termination of stress below $65 \%$ (bars indicate medians of the groups). (b) At I week after termination of chronic stress, sucrose preference was below $65 \%$ in all mice from the anhedonic group; 2 weeks after stress some mice from this group had sucrose preference below $65 \%$, while in some preference for sucrose was above this value. At 3 weeks after stress all mice from anhedonic group had preference for sucrose that was similar to that of control and nonanhedonic animals (bars indicate medians of the groups).

nonstressed controls ( 1 day after stress: $p=0.370$ and 0.499 , respectively; 1 week after stress: $p=0.801$ and 0.694 , respectively; Figures $1 \mathrm{c}$ and 3 ). It is important to note that total intake of liquid (water and sucrose solution) did not differ between the control and stress groups throughout the experiment ( $p>0.05$; Figure 1d).

Vulnerability to Develop Anhedonia is Associated with Submissive Behavior

Analysis of social behavior and sucrose consumption revealed that animals submissive in the resident-intruder test have a predisposition to stress-induced anhedonia. All eight submissive mice and only three nonsubmissive animals out of $18(16.6 \%)$ showed sucrose preference below $65 \%$ at the time point $3 \frac{1}{2}$ weeks of stress (Figure $2 a$ ). By the termination of chronic stress, six remaining mice with
Baseline $1 d$ after stress $1 w$ after stress

a

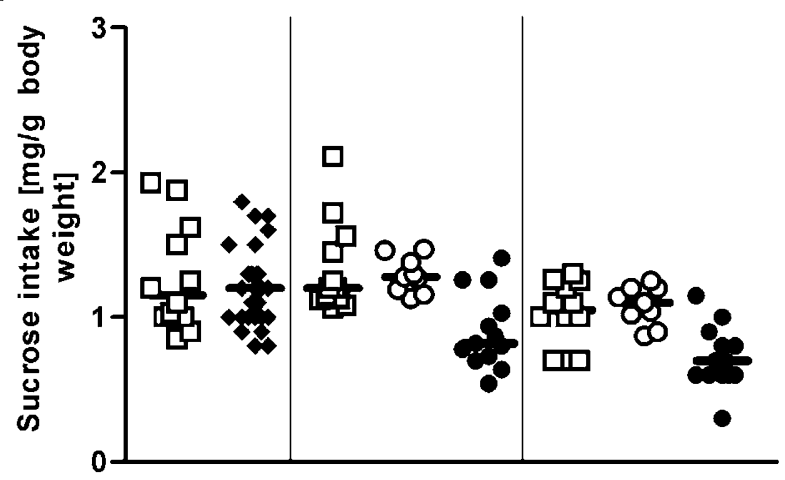

b

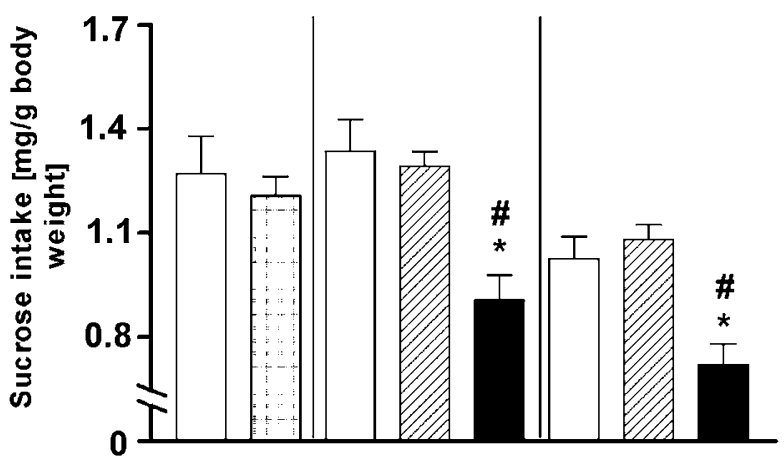

Figure 3 Chronic stress leads to a decrease in sucrose intake calculated per body weight. (a) Individual data indicate a strong decrease in intake of sucrose calculated per body weight in anhedonic mice, whereas in nonanhedonic mice this parameter was similar to control values (bars indicate medians of the groups; groups are marked as in Figure I). (b) After stress, mean intake of sucrose calculated per body weight in mice defined as anhedonic was significantly lower than in control and nonanhedonic groups (* $p<0.05$ vs control group and ${ }^{\#} p<0.05$ vs nonanhedonic group, groups are marked as in Figure I).

submissive behavior and seven of 18 (38.9\%) nonsubmissive mice were characterized as anhedonic (Figure 2a; note that two submissive animals died by this time point). The percentage of nonsubmissive animals vulnerable to anhedonia was significantly lower in comparison to submissive group at both time points $(p=0.002$ and 0.0461 , respectively; Fischer's exact test).

Thus, all mice with a submissive behavior developed anhedonia, and none of nonanhedonic individuals showed submissive behavior. Moreover, those nonsubmissive mice that displayed anhedonia exhibited nonsignificantly longer latencies to attack (Figure $4 \mathrm{~b}$ ) and significantly diminished numbers of attacks $(p=0.041$; Figure $4 c)$, that is, showed less aggressive behavior in situation of social conflict, when compared to mice that were nonanhedonic.

\section{Behavioral Analysis of Anhedonic and Nonanhedonic Mice}

Analysis of behavior studied in baseline conditions (before stress) did not reveal any differences between anhedonic and nonanhedonic groups in open field, novel cage, new object exploration, O-maze, and dark/light box (see Table 1). 


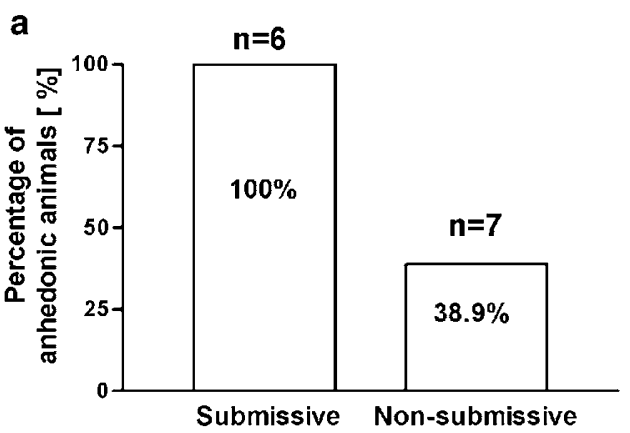

b

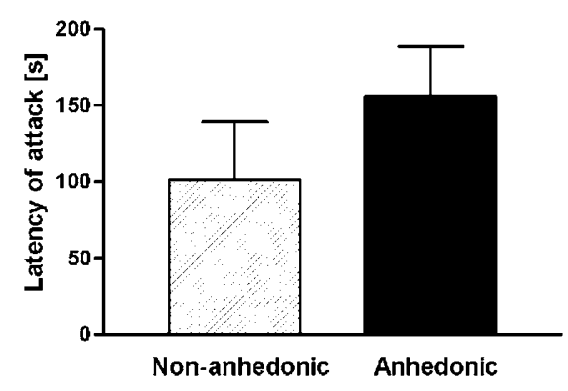

C

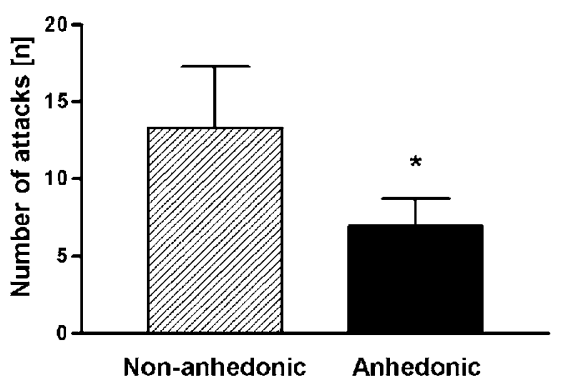

Figure 4 Vulnerability to stress-induced anhedonia is related to submissive behavior. (a) Analysis of stressed mice with previously defined social rank show that all submissive mice developed anhedonia. In contrast, only seven out of 18 nonsubmissive individuals were defined as anhedonic after termination of stress procedure (number of animals is listed above the columns). (b) The mean latency of attack of nonsubmissive mice from the anhedonic group was nonsignificantly longer when compared with mice nonanhedonic. (c) The mean number of attacks of nonsubmissive mice from the anhedonic group was significantly lower than in mice nonanhedonic (*p $<0.05$ vs nonanhedonic group).

During the first week after termination of the stress procedure, when all anhedonic mice showed a stable decrease of sucrose preference, anhedonic, nonanhedonic, and nonstressed mice were simultaneously tested in a battery of behavioral tests. At this time point, anhedonic group consisted of 13 mice (six submissive and seven nonsubmissive), nonanhedonic group consisted of nine mice (all nonsubmissive; note that two nonsubmissive animals died after the termination of stress procedure), and control group did not change.

In the forced swim test, anhedonic mice exhibited a pronounced decrease of the latency to begin floating compared to control and nonanhedonic animals $(p<0.001$ and $p=0.001$, respectively; Figure $5 \mathrm{a}$ and $\mathrm{b}$ ). The total time spent floating was significantly elevated in anhedonic mice vs control and nonanhedonic groups $(p<0.001$ and $p=0.011$, respectively; Figure $5 c$ and $d)$. There was no significant difference in latency to begin floating and total time spent floating between nonanhedonic and control groups ( $p=0.915$ and 0.618 , respectively). Thus, nonanhedonic animals and nonstressed control mice exhibited similar behavior in this test, indicating that the increase of floating behavior in chronically stressed animals is related to presence of anhedonic status.

In the new object exploration paradigm, anhedonic mice displayed decreased duration of new object exploration compared to control and nonanhedonic mice $(p=0.043$ and 0.045 , respectively; Figure $6 a$ and $b)$. In the novel cage, anhedonic animals revealed a decreased number of exploratory rearings $v s$ nonstressed controls and nonanhedonic animals $(p=0.004$ and 0.023 , respectively; Figure $6 c$ and $d)$. Parameters of exploratory behavior of nonanhedonic mice in the new object exploration and novel cage tests were not significantly different from controls ( $p=0.729$ and 0.067 , respectively). Both tests provide evidence that decreased exploratory activity in stressed mice is associated with the presence of anhedonia.

In two anxiety paradigms, anhedonic and nonanhedonic mice showed similar behavioral changes, spending less time in the open arms of the O-maze and in the lit compartment of the dark/light box in comparison to control mice (for Omaze: $p=0.001$ and 0.02 , respectively; for dark/light box: $p=0.027$ and 0.034 , respectively; Figure 7$)$. Both anhedonic and nonanhedonic groups had decreased numbers of exits to the anxiety-related compartments $v s$ control (for O-maze: $p=0.001$ and 0.002 , respectively; for dark/light box: $p=0.029$ and 0.033, respectively; Figure $7 c$ and $d$ ). Anhedonic and nonanhedonic mice showed a very similar behavior in both tests, indicating that elevated anxiety is a consequence of chronic stress, which is not related to hedonic status.

In the open field test, both anhedonic and nonanhedonic mice showed increased mean total distance moved as compared to control animals when the open field was illuminated with light of $25 \mathrm{~lx} \quad(p=0.014$ and 0.012 , respectively; Figure 8a). This finding indicates that chronic stress leads to hyperactivity in mice, when tested under light of modest brightness, which may be a subtle stressor for the animals. When the illumination strength in the open field was reduced to $5 \mathrm{~lx}$, no hyperactivity in stressed mice was observed (Figure 8b). Anhedonic and nonanhedonic animals did not differ in either protocol of the open field test in mean total distance moved $(p>0.05)$. When locomotor activity was studied using activity boxes in complete darkness, anhedonic and nonanhedonic mice again showed similar values $(p=0.496)$. However, the total distance moved of both anhedonic and nonanhedonic groups was significantly decreased compared to the nonstressed control group ( $p=0.028$ and 0.032 , respectively; Figure $8 c$ ).

The body weight of both anhedonic and nonanhedonic groups was reduced compared to the nonstressed control group ( $p=0.013$ and 0.010 , respectively; Figure 9 ). There was no significant difference between anhedonic and nonanhedonic mice $(p=0.330)$.

\section{DISCUSSION}

In the present chronic stress protocol, a decrease of preference for sucrose occurred in a subgroup of stress- 
a

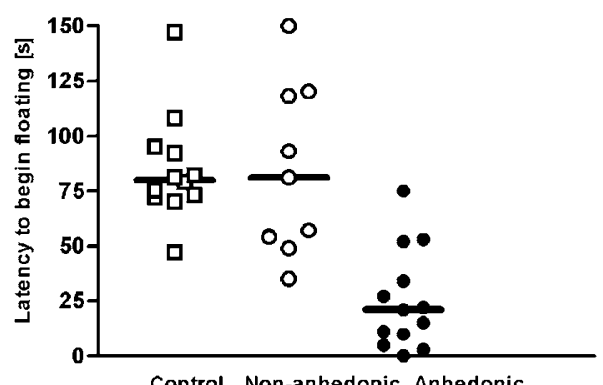

b

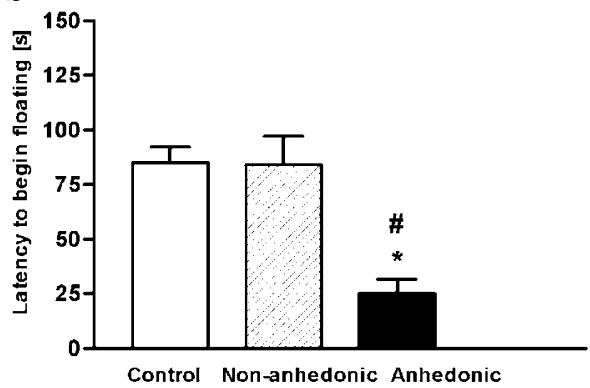

C

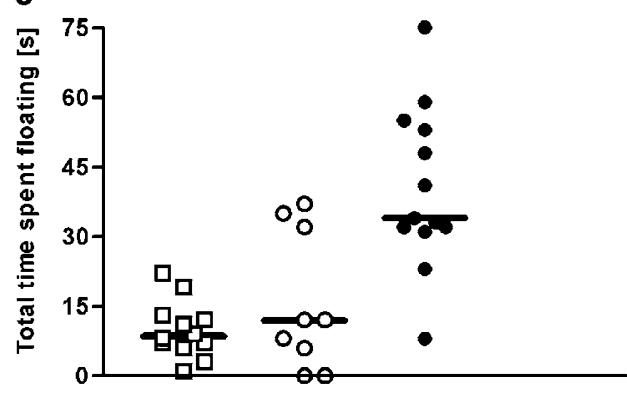

Control Non-anhedonic Anhedonic

d

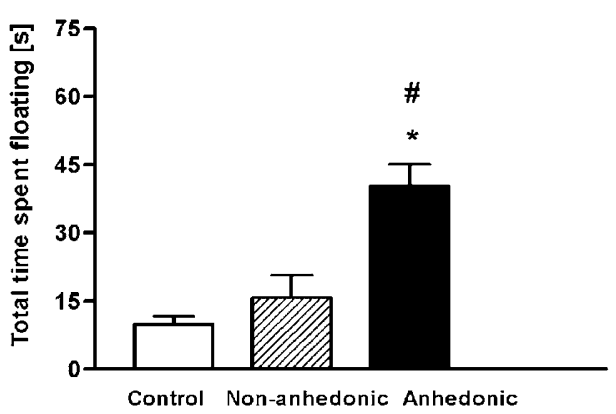

Figure 5 Increase of floating in the forced swim test is associated with anhedonia, but not with chronic stress alone. (a) Individual data indicate a strong decrease of latency to begin floating in anhedonic mice, whereas behavior of nonanhedonic mice was similar to controls (bars indicate medians of the groups). (b) The mean latency to begin floating in the anhedonic group was significantly decreased $(* p<0.05$ vs control group and ${ }^{\#} p<0.05$ vs nonanhedonic group). (c) Individual data show a largely increased total time spent floating in anhedonic mice, while behavior of nonanhedonic mice was similar to control (bars indicate medians of the groups). (d) Mean total time spent floating in the anhedonic group was significantly increased $\left({ }^{*} p<0.05\right.$ vs control group and ${ }^{*} p<0.05$ vs nonanhedonic group). There were no significant differences between nonanhedonic and control groups in any of the parameters $(p>0.05)$.

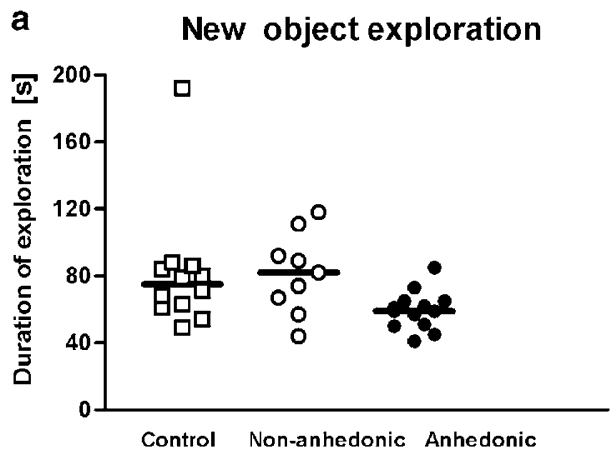

b
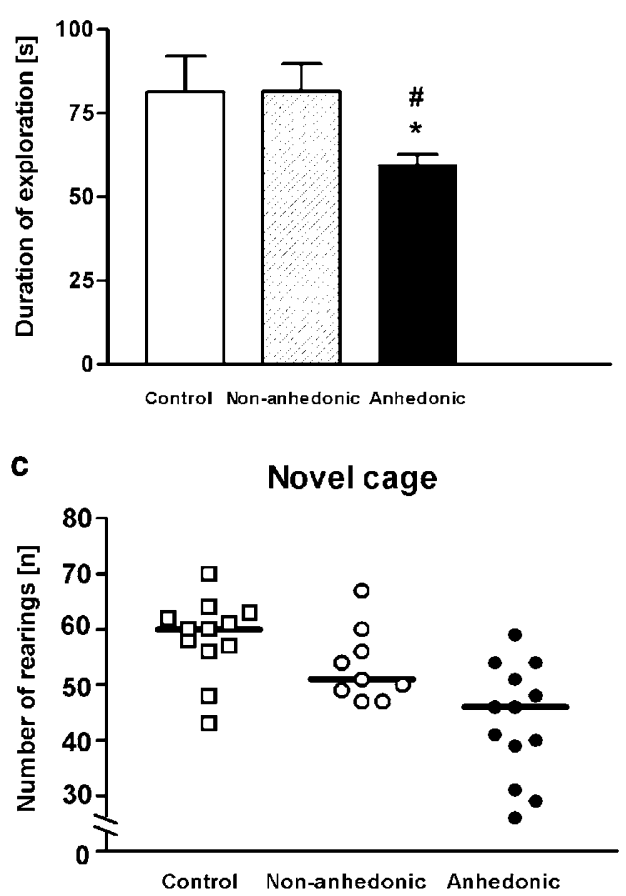

d

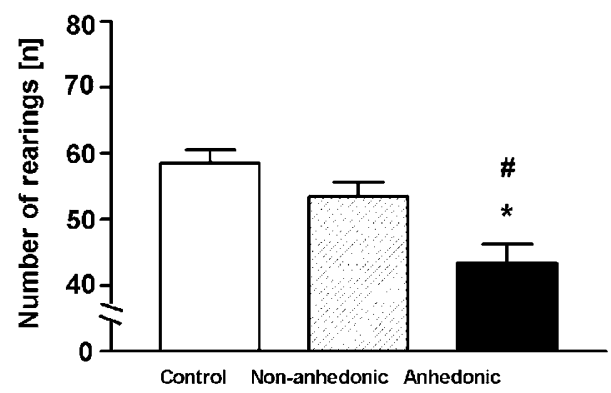

Figure 6 Decreased exploratory behavior is associated with anhedonia, but not with chronic stress alone. (a) Individual data show a decreased duration of exploration in anhedonic mice in the new object exploration test (bars indicate medians of the groups). (b) The mean of duration of exploration was significantly decreased in the anhedonic group $(* p<0.05$ vs control group and ${ }^{\#} p<0.05$ vs nonanhedonic group), but not in the nonanhedonic group ( $p>0.05$ vs control group). (c) Individual data show a decreased number of exploratory rearings of anhedonic mice in the novel cage test (bars indicate medians of the groups). (d) The mean number of rearings was significantly decreased in the anhedonic group (*p $<0.05$ vs control group and ${ }^{\#} p<0.05$ vs nonanhedonic group), but not in the nonanhedonic group ( $p>0.05$ vs control group). 
a

O-maze

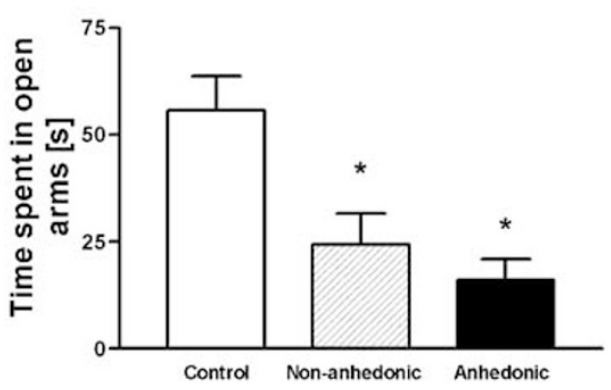

b

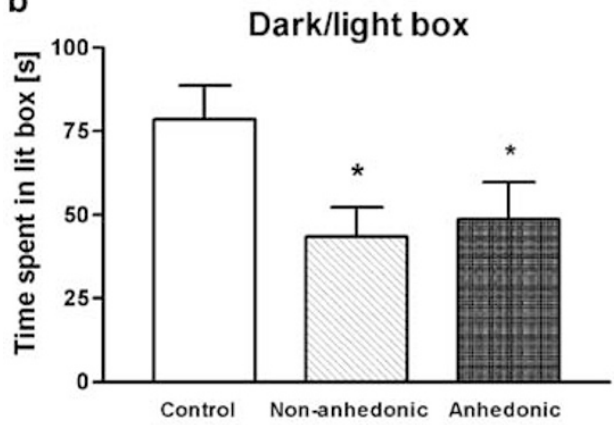

C

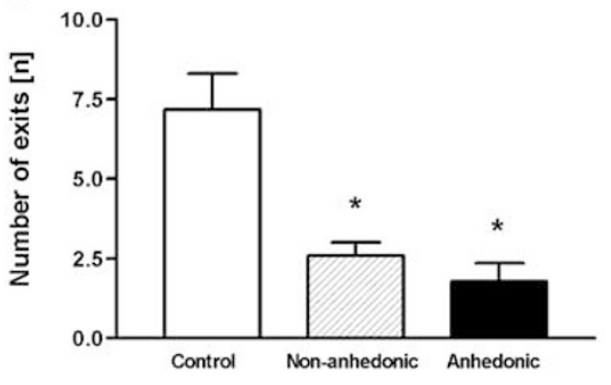

d

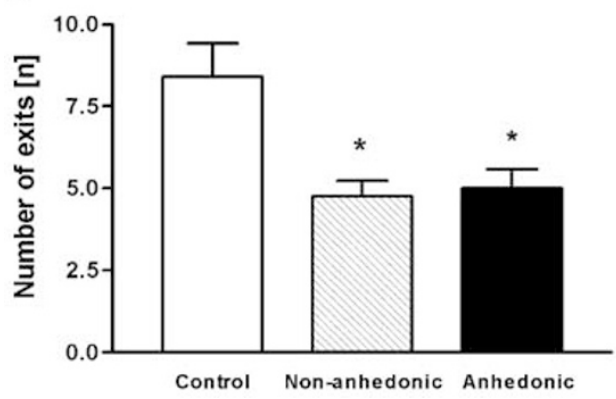

Figure 7 Chronic stress results in elevated anxiety that occurs independently from anhedonia. (a,b) The mean time spent in the open arms in the O-maze and in the lit compartment of the dark/light box were significantly decreased both in anhedonic and nonanhedonic mice when compared to controls (*p $<0.05$ vs control group). (c, d) Mean numbers of exits to the open arms in O-maze and to the lit compartment of the dark light box were significantly reduced in anhedonic and nonanhedonic groups as compared to controls (* $p<0.05$ vs control group).

exposed mice that we categorized as anhedonic. However, another subgroup of stress-exposed mice did not exhibit a decrease of sucrose preference and was therefore defined as nonanhedonic. Since these animals were exposed to the same extent of stress as those mice that developed

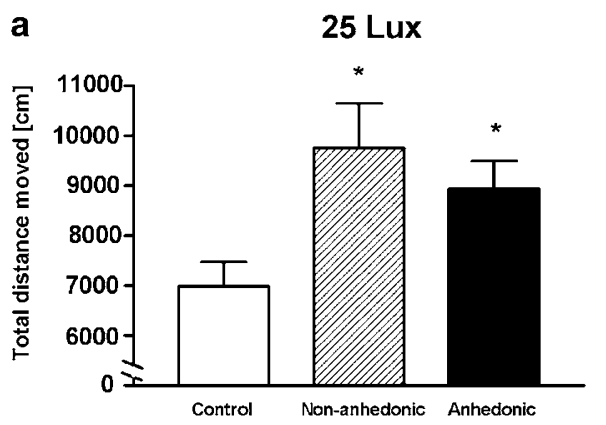

b

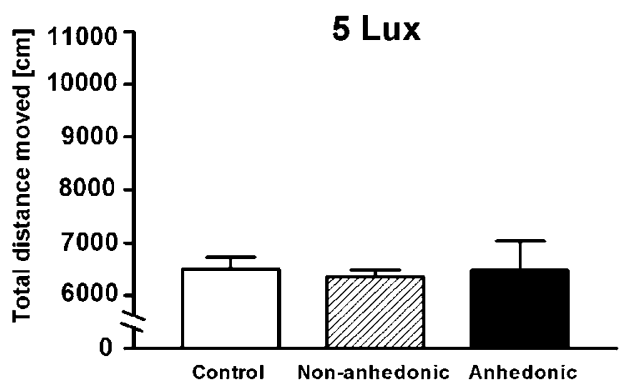

C

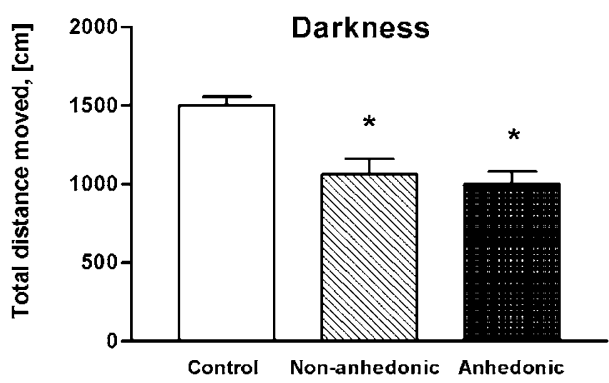

Figure 8 Chronic stress leads to locomotor disturbances that are not related to anhedonia. (a) Mean total distance moved was significantly increased in anhedonic and nonanhedonic mice in the open field lit with light of $25 \mathrm{l} x$ intensity as compared to the controls (* $p<0.05$ vs control group). (b) The mean total distance moved in the open field did not differ between nonanhedonic, anhedonic, and control mice when the illumination strength was reduced to $5 \mathrm{~lx}(p>0.05)$. (c) In activity boxes test, performed in darkness, the mean distance moved was significantly lower both in anhedonic and nonanhedonic mice as compared to controls (* $p<0.05$ vs control group).

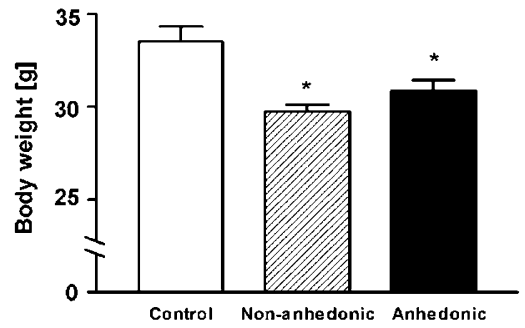

Figure 9 Stress-induced reduction of body weight is similar in anhedonic and nonanhedonic mice. The means of body weight were significantly decreased in both anhedonic and nonanhedonic groups as compared to controls (* $p<0.05$ vs control group). There was no significant difference between anhedonic and nonanhedonic mice ( $p>0.05)$.

anhedonia, they were taken as an internal control for stress-induced effects not associated with hedonic deficit. The behavioral analysis of both groups revealed that 
anhedonia in stressed mice is accompanied by other features of depressive-like behavior, such as increased floating in the forced swim test and decreased exploration of novelty. In contrast, increased anxiety, changed locomotor activity, and loss of body weight are the consequences of chronic stress that occur independently from the development of anhedonia. Importantly, baseline behavior in anhedonic, nonanhedonic, and control groups of mice did not differ before stress. Thus, stress-induced anhedonia is associated with behavioral features that cannot be seen in stressed mice without hedonic deficit. Interestingly, predisposition for stress-induced anhedonia was associated with submissive behavior in a resident-intruder test. With respect to the type of social behavior, all submissive mice, as well as a minority of nonsubmissive animals developed anhedonia.

\section{Definition of Hedonic Status in Sucrose Test}

A decrease of sucrose consumption in a limited access paradigm has been used previously to determine hedonic deficits. However, absolute intake of sucrose solution within a short period of time (eg $2 \mathrm{~h}$ ) can be influenced by many factors not related to the hedonic state. Thus, sucrose consumption can be disturbed by subtle stressors occurring during the sucrose test and by prolonged effects of acutely applied stress procedures (Pucilowski et al, 1993), food and water deprivation (Forbes et al, 1996; Reid et al, 1997), as well as by reduced metabolic needs in sucrose due to the loss of body weight (Matthews et al, 1995). In conclusion, demonstration of anhedonia by fluid intake is not regarded as a convincing measure (Nestler et al, 2003).

Therefore, we modified the protocol of the sucrose test and evaluated sucrose preference, calculated as a percentage of consumed sucrose solution of the total amount of liquid drunk. In addition, we estimated intake of absolute amounts of sucrose solution and water and consumption of sucrose calculated per gram body weight. To minimize the influence of metabolic factors and to reduce acute stress components during the sucrose test, we did not apply food and water deprivation in our experiment. We further increased the accuracy of measurement by prolonging free access to sucrose up to $24 \mathrm{~h}$. This is of particular importance since mice usually consume small amounts of liquids and the error of measurement is high. Another reason for test prolongation was to minimize potential neophobic reactions against unusual drinking situation and taste of sucrose solution. We also attempted to balance the effect of side preference in drinking behavior by switching the bottles in the middle of the test, which was equilibrated with respect to the circadian cycle. In the end, mice had preferable and nonpreferable solution on each side of the cage during equal periods of active and inactive phases, which was reported to be of importance for evaluation of drinking behavior in sucrose test (Kant and Bauman, 1993).

In the present study, a decrease in preference for sucrose below $65 \%$ was taken as a criterion for anhedonia. In earlier studies, mice and rats with a sucrose preference below $65 \%$ showed an increased threshold of intracranial self-stimulation (Moreau et al, 1992) and a decreased latency and increased duration of REM sleep (Vogel et al, 1990; Moreau et al, 1995; Cheeta et al, 1997), which are considered as physiological correlates of a depressive syndrome in rodents. Furthermore, a chronic stress induced decrease of sucrose preference below $65 \%$ was also associated with a reduction of sexual activity (D'Aquila et al, 1994; Brotto et al, 2001) and alterations of circadian rhythms (Stewart et al, 1990; Solberg et al, 1999). In our study, pronounced differences occurred in depressive-like behavior between groups of mice assigned to anhedonic and nonanhedonic according to this criterion.

\section{Behavioral Changes in Stressed Mice with and without Anhedonia}

As our chronic stress protocol leads to anhedonia in the majority but not all animals, we could analyze whether stress-induced anhedonia is associated with specific behaviors as compared to effects of stress without hedonic deficit. The group of anhedonic mice revealed a decrease of latency to begin floating in the forced swim test and an increase in the total time spent floating. Nonanhedonic mice showed similar scores of floating as nonstressed controls. Thus, increased floating in mice exposed to chronic stress is related to the presence of hedonic deficit.

Two exploratory tests revealed a significantly decreased exploration of a novel environment and of a novel object in anhedonic mice, as compared to control and nonanhedonic group. Thus, decreased interest to novelty in mice exposed to chronic stress is related to the presence of hedonic deficit. Since both anhedonic and nonanhedonic animals displayed similar anxiety-related behavior and locomotion under the lighting of the same intensity as employed in exploration tasks, it is very unlikely that the decrease of exploration behavior was due to changes in anxiety or locomotion.

Both anhedonic and nonanhedonic mice displayed increased anxiety when compared to nonstressed controls, suggesting that elevated anxiety is a consequence of chronic stress, and is not related to the development of anhedonia. To our knowledge, this is the first experimental evidence in rodents that separates anxiety from behaviors related to anhedonia. Notably, the changes in behavior only related to stress as well as the decrease in body weight were similarly pronounced in anhedonic and nonanhedonic animals, suggesting that the stress exposure was perceived as equally stressful by both groups. Thus, mice, which were nonanhedonic served as an adequate internal control for stressinduced behavioral features not associated with hedonic state.

\section{Individual Vulnerability to Anhedonia and Social Behavior}

The predisposition to stress-induced anhedonia was indicated by social behavior as shown by the residentintruder test. Submissive individuals have been found in different paradigms and ethological studies to share features of helpless or defeated animals in behavioral models of depression, such as diminished sexual behavior and exploration, reduced social interactions, and hypolocomotion (Raab et al, 1986; Pare, 2000; Vollmayr and Henn, 2001). Moreover, passive coping and submissive social behavior are related to enhanced behavioral and neuroen- 
docrine responses to stress in rodents (De Boer et al, 1990; Pare and Redei, 1993; Blanchard et al, 1993; Strekalova, 1995; D’Amato et al, 2001). Our finding presented here is consistent with this view.

In summary, we show that employed chronic stress induces in a subgroup of mice a depression-like syndrome characterized by decreased preference for sucrose, increased floating in forced swimming, and decreased exploration of novelty. Other behavioral changes such as elevated anxiety and locomotor disturbances are found in both anhedonic and anhedonia nonanhedonic mice, indicating that these behavioral features occur independent of the presence of the anhedonic state. Importantly, our approach allows distinguish factors that correlate specifically with stress-induced anhedonia from those that are a consequence of the chronic stress alone.

\section{ACKNOWLEDGEMENTS}

We acknowledge technical assistance of $\mathrm{B}$ Cline, $\mathrm{S}$ Chourbaji, M Knyssok, S Koch, M Motika, G Sadovska, and $\mathrm{V}$ Vengeliene. This work was supported by a grant from the Deutsche Forschungsgemeinschaft (GA 427/4-1 to PG).

\section{REFERENCES}

Blanchard DC, Sakai RR, McEwen B, Weiss SM, Blanchard RJ (1993). Subordination stress: behavioral, brain, and neuroendocrine correlates. Behav Brain Res 58: 113-121.

Brotto LA, Gorzalka BB, LaMarre AK (2001). Melatonin protects against the effects of chronic stress on sexual behaviour in male rats. Neuroreport 16: 3465-3469.

Cheeta S, Ruigt G, van Proosdij J, Willner P (1997). Changes in sleep architecture following chronic mild stress. Biol Psychiatry 41: 419-427.

D’Amato FR, Rizzi R, Moles A (2001). A model of social stress in non-submissive mice: effects on sociosexual behaviour. Physiol Behav 73: 421-426.

D’Aquila PS, Brain P, Willner P (1994). Effects of chronic mild stress on performance in behavioural tests relevant to anxiety and depression. Physiol Behav 56: 861-867.

D’Aquila PS, Monleon S, Borsini F, Brain P, Willner P (1997). Antianhedonic actions of the novel serotonergic agent flibanserin, a potential rapidly-acting antidepressant. Eur J Pharmacol 340: 121-132.

De Boer SF, Slangen JL, Van der Gugten J (1990). Plasma catecholamine and corticosterone levels during active and passive shock-prod avoidance behavior in rats: effects of chlordiazepoxide. Physiol Behav 47: 1089-1098.

Forbes NF, Stewart CA, Matthews K, Reid IC (1996). Chronic mild stress and sucrose consumption: validity as a model of depression. Physiol Behav 60: 1481-1484.

Hamilton M (1967). Development of a rating scale for primary depressive illness. Br J Soc Clin Psychol 6: 278-296.

Harkin A, Houlihan DD, Kelly JP (2002). Reduction in preference for saccharin by repeated unpredictable stress in mice and its prevention by imipramine. J Psychopharmacol 16: 115-123.

Harris RB, Zhou J, Youngblood BD, Smagin GN, Ryan DH (1997). Failure to change exploration or saccharin preference in rats exposed to chronic mild stress. Physiol Behav 63: 91-100.

Hata T, Itoh E, Nishikawa H (1995). Behavioral characteristics of SART-stressed mice in the forced swim test and drug action. Pharmacol Biochem Behav 51: 849-853.
Henn FA (1980). Suicide risk with tryclicic antidepressants. Am Fam Physician 21: 1-23.

Kant GJ, Bauman RA (1993). Effects of chronic stress and time of day on preference for sucrose. Physiol Behav 54: 499-502.

Katz RJ (1981). Animal models and human depressive disorders. Neurosci Biobehav Rev 5: 231-246.

Katz RJ (1982). Animal model of depression: pharmacological sensitivity of a hedonic deficit. Pharmacol Biochem Behav 16 965-968.

Kessler RC, McGonagle KA, Zhao S, Nelson CB, Hughes M, Eshleman $S$ et al (1994). Lifetime and 12-month prevalence of DSM-III-R psychiatric disorders in the United States. Results from the National Comorbidity Survey. Arch Gen Psychiatry 51: 8-19.

Klein DF (1974). Endogenomorphic depression. A conceptual and terminological revision. Arch Gen Psychiatry 31: 447-454.

Krsiak M (1975). Timid singly-housed mice: their value in prediction of psychotropic activity of drugs. Br J Pharmacol 55: 141-150.

Krsiak M, Podhorna J, Miczek KA (1998). Aggressive and social behavior after alprazolam withdrawal: experimental therapy with Ro 19-8022. Neurosci Biobehav Rev 23: 155-161.

Kudryavtseva NN, Bakshtanovskaya IV, Koryakina LA (1991). Social model of depression in mice of C57BL/6J strain. Pharmacol Biochem Behav 38: 315-320.

Matthews K, Forbes N, Reid IC (1995). Sucrose consumption as an hedonic measure following chronic unpredictable mild stress. Physiol Behav 57: 241-248.

Moreau JL, Jenck F, Martin JR, Mortas P, Haefely WE (1992). Antidepressant treatment prevents chronic unpredictable mild stress-induced anhedonia as assessed by ventral tegmentum self-stimulation behavior in rats. Eur Neuropsychopharmacol 2: 43-49.

Moreau JL, Scherschlicht R, Jenck F, Martin JR (1995). Chronic mild stress-induced anhedonia model of depression; sleep abnormalities and curative effects of electroshock treatment. Behav Pharmacol 6: 682-687.

Nestler EJ, Gould E, Manji H, Buncan M, Duman RS, Greshenfeld HK et al (2003). Preclinical models: status of basic research in depression. Biol Psychiatry 52: 503-528.

Pare WP (2000). Investigatory behavior of a novel conspecific by Wistar Kyoto, Wistar and Sprague-Dawley rats. Brain Res Bull 53: 759-765.

Pare WP, Redei E (1993). Depressive behavior and stress ulcer in Wistar Kyoto rats. J Physiol Paris 87: 229-238.

Pucilowski O, Overstreet DH, Rezvani AH, Janowsky DS (1993). Chronic mild stress-induced anhedonia: greater effect in a genetic rat model of depression. Physiol Behav 54: $1215-1220$.

Raab A, Dantzer R, Michaud B, Mormede P, Taghzouti K, Simon H et al (1986). Behavioural, physiological and immunological consequences of social status and aggression in chronically coexisting resident-intruder dyads of male rats. Physiol Behav 36: 223-228.

Reid I, Forbes N, Stewart C, Matthews K (1997). Chronic mild stress and depressive disorder: a useful new model? Psychopharmacology (Berl) 134: 365-567.

Solberg LC, Horton TH, Turek FW (1999). Circadian rhythms and depression: effects of exercise in an animal model. Am J Physiol 276: R152-R161.

Stewart KT, Rosenwasser AM, Hauser H, Volpicelli JR, Adler NT (1990). Circadian rhythmicity and behavioral depression: I. Effects of stress. Physiol Behav 48: 149-155.

Strekalova TV (1995). The characteristics of the defensive behavior of rats in accordance with their resistance to emotional stress. Zh Vyssh Nerv Deiat Im I P Pavlova 45: 420-422.

Vogel G, Neill D, Hagler M, Kors D (1990). A new animal model of endogenous depression: a summary of present findings. Neurosci Biobehav Rev 14: 85-91. 
Vollmayr B, Henn FA (2001). Learned helplessness in the rat: improvements in validity and reliability. Brain Res Brain Res Protoc 8: 1-7.

Willner P (1991). Animal models as simulations of depression. Trends Pharmacol Sci 12: 131-136.

Willner P (1997). Validity, reliability and utility of the chronic mild stress model of depression: a 10-year review and evaluation. Psychopharmacology 134: 319-329.
Willner P, D’Aquila PS, Coventry T, Brain P (1995). Loss of social status: preliminary evaluation of a novel animal model of depression. J Psychopharmacol 9: 207-213.

Willner P, Towell A, Sampson D, Sophokleous S, Muscat R (1987). Reduction of sucrose preference by chronic unpredictable mild stress, and its restoration by a tricyclic antidepressant. Psychopharmacology (Berl) 93: 358-364. 